\title{
A Randomized Trial Among Compression Plus Nonsteroidal Antiinflammatory Drugs, Aspiration, and Aspiration With Steroid Injection for Nonseptic Olecranon Bursitis
}

\author{
Joon Yub Kim MD, PhD, Seok Won Chung MD, PhD, Joo Hak Kim MD, \\ Jae Hong Jung MD, Gwang Young Sung MD, Kyung-Soo Oh MD, \\ Jong Soo Lee MD
}

Received: 12 July 2015/ Accepted: 29 September 2015/Published online: 13 October 2015

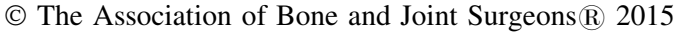

\begin{abstract}
Background Olecranon bursitis might be a minor problem in the outpatient clinic but relatively be common to occur. However, there are few well-designed studies comparing approaches to treatment.

Questions/purposes (1) Which treatment (compression bandaging with nonsteroidal antiinflammatory drugs [NSAIDs], aspiration, or aspiration with steroid injections) is associated with the highest likelihood of resolution of nonseptic olecranon bursitis? (2) Which treatment is

Each author certifies that he or she, or a member of his or her immediate family, has no funding or commercial associations (eg, consultancies, stock ownership, equity interest, patent/licensing arrangements, etc) that might pose a conflict of interest in connection with the submitted article.

All ICMJE Conflict of Interest Forms for authors and Clinical Orthopaedics and Related Research ${ }^{\mathbb{R}}$ editors and board members are on file with the publication and can be viewed on request.

Clinical Orthopaedics and Related Research ${ }^{\mathbb{R}}$ neither advocates nor endorses the use of any treatment, drug, or device. Readers are encouraged to always seek additional information, including FDAapproval status, of any drug or device prior to clinical use.

Each author certifies that his or her institution approved the human protocol for this investigation, that all investigations were conducted in conformity with ethical principles of research, and that informed consent for participation in the study was obtained.

This work was performed at Seonam University College of Medicine Myongji Hospital, Goyang-si, Korea, and Konkuk University School of Medicine, Seoul, Korea.
\end{abstract}

J. Y. Kim, J. H. Kim, J. H. Jung, G. Y. Sung

Department of Orthopedic Surgery, Seonam University College

of Medicine Myongii Hospital, Goyang-si, Korea

S. W. Chung ( $\square)$, K.-S. Oh, J. S. Lee

Department of Orthopaedic Surgery, Konkuk University School

of Medicine, 120-1 Neungdong-ro (Hwayang-dong),

Gwangjin-gu, Seoul 143-729, Korea

e-mail: smilecsw@gmail.com associated with earliest resolution of symptoms? (3) What factors are associated with treatment failure by 4 weeks? Methods We enrolled 133 patients from two centers; after applying prespecified exclusions (septic bursitis or concomitant inflammatory arthritis, intraarticular elbow pathology, recent aspiration or steroid injection done elsewhere, and refusal to participate), 90 patients were randomly allocated to receive compression bandaging with NSAIDs (C), aspiration (A), or aspiration with steroid injection (AS) groups (30 patients in each). The groups were similar at baseline in terms of age and gender. Seven patients (four from Group A and three from Group AS) were lost to followup. All patients were followed up weekly for 4 weeks, and the same treatment procedure was repeated if the bursitis recurred with any substantial fluid collection. At 4 weeks, the state of resolution and pain visual analog scale (VAS) were evaluated. Failed resolution was defined as presence of persistent olecranon bursal fluid collection at Week 4 after the initiation of the treatment; on the contrary, if bursal fluid collection was clinically reduced or completely disappeared by the end of Week 4, the treatment was considered successful. We compared the proportion of resolution by Week 4 and the median times to resolution among the treatment groups. In addition, we evaluated whether the resolution affected pain VAS and what factors were associated with the resolution. Results There were no differences in the proportion of patients whose bursitis resolved by Week 4 among the three treatment groups (Group C: 25 of 30 [83\%], relative risk of resolution failure: 0.68 [95\% confidence interval $\{\mathrm{CI}\}, 0.27-1.72], \mathrm{p}=0.580$; Group A: 17 of 26 [65\%], relative risk of resolution failure: 2.19 [95\% CI, 0.984.87], $p=0.083$; Group AS: 23 of 27 [85\%], relative risk of resolution failure: 0.59 [95\% CI, 0.22-1.63], $\mathrm{p}=0.398)(\mathrm{p}$ $=0.073$ ). Steroid injection after aspiration (Group AS) was 
associated with the earliest resolution (2.3 weeks [range, 14 weeks]) when compared with aspiration alone (Group A; 3.1 weeks [range, 2-4 weeks]) and compression bandaging with NSAIDs (Group C; 3.2 weeks [range, 2-4 weeks]), p $=0.015)$. Longer duration of symptoms before treatment was the only factor associated with treatment failure by 4 weeks (failed resolution: 6 weeks [range, 2-9 weeks]; successful resolution: 4 weeks [range, 0.4-6 weeks]; $\mathrm{p}=$ 0.008).

Conclusions With the numbers available, there were no differences in efficacy when compression bandaging with NSAIDs, aspiration, and aspiration with steroid injection were compared. However, we were powered only to detect a $30 \%$ difference, meaning that if there were a smaller difference in efficacy among the groups, we might not have detected it in a study of this size. Our data can be used as pilot data to power future prospective (and likely multicenter) trials. Because olecranon bursitis can recur, and because treatments like aspiration and aspiration with steroid injection can cause complications, unless future trials demonstrate clear efficacy advantages of aspiration and/or injection both at short and longer terms, we suggest that compression bandaging and a short course of NSAIDs may offer the most appropriate balance of safety and efficacy.

Level of Evidence Level II, therapeutic study.

\section{Introduction}

Olecranon bursitis is characterized by fluid accumulation in the bursa with or without inflammation $[5,11]$. It is relatively common with a reported minimum annual incidence of up to 10 per 100,000 [6, 9]. Treatment options for nonseptic olecranon bursitis include compression bandaging with nonsteroidal antiinflammatory drugs (NSAIDs), needle aspiration of the bursal fluid, needle aspiration with steroid injection, or surgical management such as open or arthroscopic bursectomy, osseous resection, and percutaneous suction drainage $[1,6,15]$. Among these, the nonsurgical options rather than surgical options are more commonly used in clinical practice and preferred in the initial treatment of the nonseptic olecranon bursitis [15]. However, the resolution failure of olecranon bursitis after the initial treatment is not uncommon and a challenge for orthopaedic surgeons. In addition, there has been no standardized treatment for nonseptic olecranon bursitis and the treatment approach largely depends on each surgeon's preferences and experience [18].

To the authors' knowledge, there are few studies comparing the results according to the treatment methods for nonseptic olecranon bursitis $[13,20]$. The establishment of an evidence-based treatment guideline for nonseptic olecranon bursitis would be an important step, but data from randomized trials should support any such guidelines [15]. To our knowledge, there has been only one randomized controlled study by Smith et al. [17]; however, their study did not involve the simple aspiration as a treatment method and the sample size was relatively small with only 42 cases. Therefore, we designed a randomized prospective controlled trial to compare three treatments for nonseptic olecranon bursitis: compression bandaging with NSAIDs (compression/NSAIDs), aspiration, and aspiration with steroid injection. We asked the following questions: (1) Which treatment (compression bandaging with NSAIDs, aspiration, or aspiration with steroid injection) is associated with the highest likelihood of resolution of nonseptic olecranon bursitis? (2) Which treatment is associated with earliest resolution of symptoms among successful treatments? (3) What factors are associated with treatment failure by 4 weeks?

\section{Patients and Methods}

We conducted this randomized prospective controlled study in accordance with the principles of the Declaration of Helsinki. The reporting of data from this trial complies with the CONSORT statement. This study was approved by the institutional review board of the authors' hospital (No. 14-086) and registered at Clinical Research Information Service (identifier KCT0001525).

\section{Sample Size Calculation and Patient Allocation}

Sample sizes were calculated to detect a $30 \%$ difference in the proportion of patients whose olecranon bursitis resolved with treatment. The $30 \%$ difference was determined to be clinically significant based on a pilot study in the authors' hospital that included 30 patients. A sample size of 30 patients in each group was required for a power of $80 \%$ at a Type I error level of 0.05 and for an expected dropout rate of $20 \%$.

The inclusion criterion of this study was nonseptic olecranon bursitis, which was initially diagnosed by two orthopaedic surgeons who were the authors of this study (JYK, SWC). A total of 133 patients with nonseptic olecranon bursitis were prospectively enrolled from two hospitals between March 2011 and February 2015. Exclusion criteria were septic bursitis $(n=10)$, bursitis combined with gout or rheumatoid arthritis $(n=3)$, concomitant elbow pathology $(n=4)$, a history of aspiration or steroid injection at another hospital $(\mathrm{n}=10)$, allergies, intolerances or medical contraindications to NSAID use $(n=0)$, and those who refused to participate in the study $(n=16)$. In 
case of suspected septic bursitis, which showed local heat and redness accompanied by fever [4, 7] (11 cases), the diagnostic aspiration and analysis of bursal fluid were performed. Among these 11 cases, 10 were confirmed as septic bursitis and one as gout arthritis, and all were excluded from the initial enrollment by the exclusion criteria. According to the exclusion criteria, 43 patients were excluded. The remaining 90 patients were randomly allocated to the compression bandaging with NSAIDs (C), aspiration (A), and aspiration with steroid injection (AS) groups (30 patients in each). Patients were randomized with a computer-generated sequence and each patient was assigned to each treatment group by opening a sealed envelope immediately before the treatment in all patients enrolled. Among these 90 patients, seven (four from Group A and three from Group AS) were lost to followup. Among the lost seven patients to followup, five were lost to followup just after the first visit, and two were lost to followup after the second visit (one was from Group A and the other one was from Group AS). The two patients who were lost to followup after the second visit showed persistent olecranon bursitis when they visited the outpatient clinic at the second week, but they refuse to receive further treatment. Although we tried to track all the lost seven patients to followup by phone, they refuse to visit again. In addition, because we could not decide the resolution of the olecranon bursitis objectively by measuring the size, all these patients were excluded from further analyses. Accordingly, 83 patients were finally enrolled in this study (Fig. 1).

The mean age of the 83 enrolled patients was 46 years (range, 13-81 years), and there were 59 males and 24 females. Forty-one patients had a history of trauma, which was defined in this study as a direct blow to the posterior elbow within the last month. The mean symptom duration was 4 weeks (range, 1-16 weeks), and the mean followup was 12 weeks (range, 4-140 weeks). The complications were checked even after a 4-week period and which determined the duration of followup. There were 30

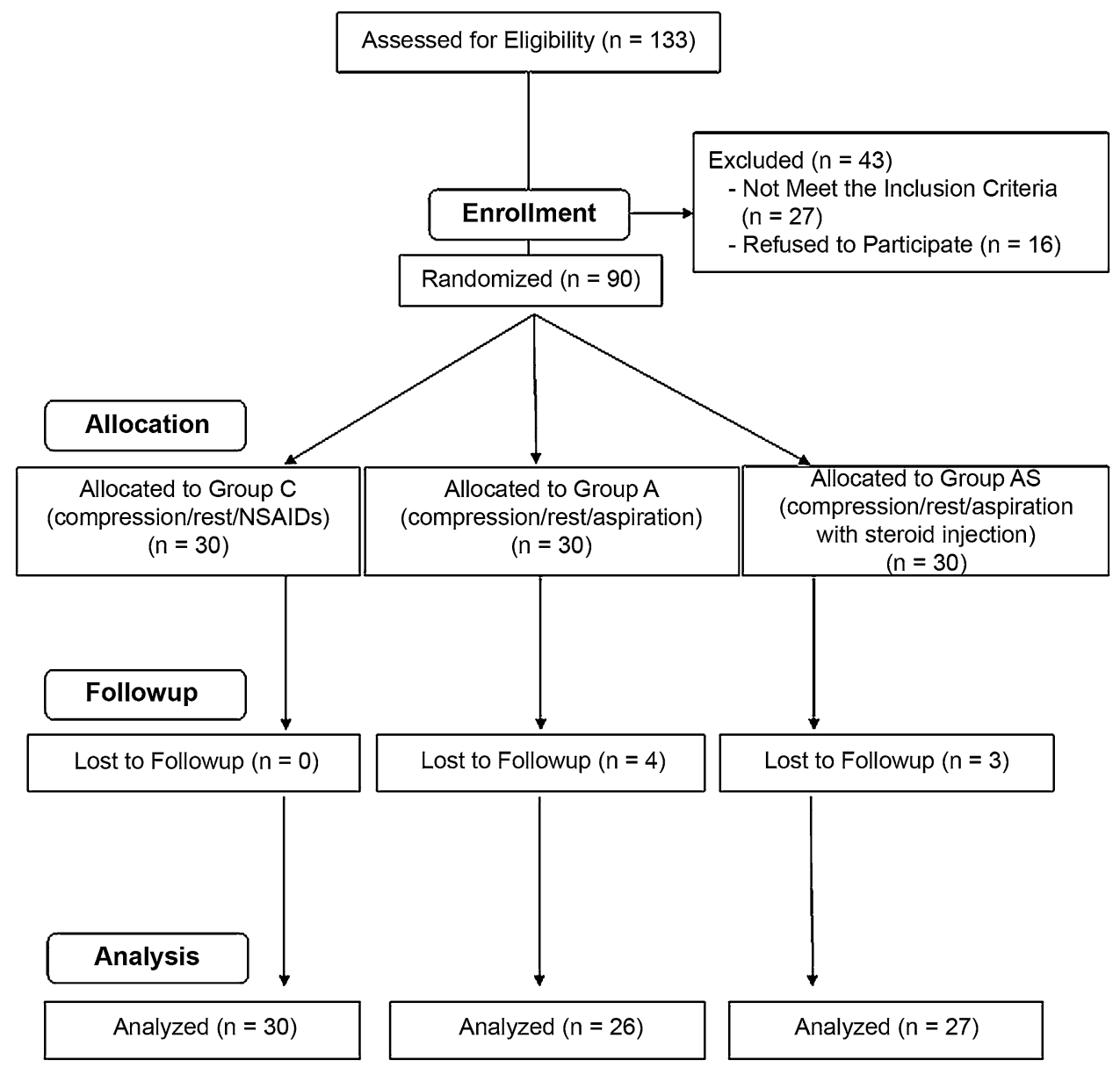

Fig. 1 The flowchart of patient allocation was elicited. One hundred thirty-three patients were initially assessed for eligibility initially, and after the exclusion process, 90 patients (30 patients in each group) were enrolled in this study. Four patients in Group A (aspiration group) and three patients in Group AS (aspiration with steroid injection) were lost to followup; therefore, 30 patients in Group C (compression with NSAIDs), 26 patients in Group A, and 27 patients in Group AS were finally analyzed. 
Table 1. Demographic data of patients

\begin{tabular}{|c|c|c|c|c|}
\hline Demographic & $\begin{array}{l}\text { Compression/NSAIDs } \\
\text { group }(\mathrm{n}=30)\end{array}$ & $\begin{array}{l}\text { Aspiration group } \\
(\mathrm{n}=26)\end{array}$ & $\begin{array}{l}\text { Aspiration with steroid } \\
\text { injection group }(n=27)\end{array}$ & $\mathrm{p}$ value \\
\hline Age (years) & 41 (range, 13-71) & 48 (range, 19-76) & 50 (range, $21-81)$ & 0.074 \\
\hline Sex (male:female) & $24: 6$ & $16: 10$ & 19:8 & 0.314 \\
\hline Dominant:nondominant hand & $22: 8$ & $19: 7$ & $20: 6$ & 0.938 \\
\hline Symptom duration (weeks) & 4 (range, 1-16) & 4 (range, $1-12$ ) & 4 (range, 1-16) & 0.986 \\
\hline Diabetes & $2 / 30$ & $5 / 26$ & $3 / 27$ & 0.355 \\
\hline Hypertension & $2 / 30$ & $8 / 26$ & $5 / 27$ & 0.065 \\
\hline Trauma history & $18 / 30$ & $13 / 26$ & $10 / 27$ & 0.223 \\
\hline
\end{tabular}

NSAIDs $=$ nonsteroidal antiinflammatory drugs.

patients in Group C, 26 patients in Group A, and 27 patients in Group AS (Table 1).

\section{Treatment Protocols}

\section{Compression Bandaging With NSAIDs Group \\ (Compression/NSAIDs, Group C)}

For the patients in Group C, only a 4-inch elastic bandage was applied to the elbow with mild compression, NSAIDs (aceclofenac [ASEC tab; Hanmi Pharm, Seoul, Korea] 100 $\mathrm{mg}$, twice a day) were prescribed for a week, and any changes to the olecranon bursitis were observed every week. The patients were asked not to rub the affected posterior elbows against hard surfaces such as desktops and floors and to reduce their physical activity as much as possible.

\section{Aspiration Group (Group A)}

The patients in Group A underwent aspiration and were followed up weekly. The aspiration was performed using a secured drape after application of povidone. An 18-gauge needle attached to a 50-cc syringe was used to puncture the bursa at a point slightly distal to its center nearly parallel to the forearm or following a slightly oblique trajectory. The needle was carefully advanced so as not to cause any additional injury or persistent drainage, and all the fluid inside the bursa was aspirated. After the aspiration, gauze was applied, and the elbow was lightly compressed by applying a 4-inch elastic bandage in the same manner as in Group C. The same NSAIDs (aceclofenac [ASEC tab; Hanmi Pharm] $100 \mathrm{mg}$, twice a day) as in Group C were prescribed for a week, and the patients were instructed to reduce their physical activity and to avoid friction of the posterior elbow region. If weekly followup revealed failed resolution of the bursitis with substantial fluid collection, aspiration was repeated following the same treatment protocol.

\section{Aspiration With Steroid Injection Group (Group AS)}

For the patients in the AS group, aspiration with steroid injection was performed in the same manner as in Group A, except for an additional injection of $1 \mathrm{~mL}$ of $40 \mathrm{mg} / \mathrm{mL}$ triamcinolone acetonide (Triam Inj; Shin Poong Pharm Co, Ltd, Ansan, Korea) mixed with $1 \mathrm{~mL}$ of $2 \%$ lidocaine (2\% Lidocaine HCL; Daihan Pharm Co, Ltd, Seoul, Korea) to the aspiration site after completing the aspiration. The patients were followed up on a weekly basis. Gauze was applied, and mild compression was performed by applying a 4-inch elastic bandage. The same NSAIDs (aceclofenac [ASEC tab; Hanmi Pharm] $100 \mathrm{mg}$, twice a day) as in Group $\mathrm{C}$ were prescribed for a week, and the same instructions were given regarding the avoidance of friction of the posterior elbow and reducing physical activity. If the bursitis failed to resolve with substantial bursal fluid collection, the aspiration and triamcinolone injection were repeated following the same treatment protocol.

Followups and Evaluations

Every patient was followed up on a weekly basis for 4 weeks after the initiation of the treatment. Resolution of the lesion and any complications related to the treatment such as infection, skin atrophy after the steroid injection, presence of persistent drainage, or chronic local pain were evaluated by two orthopaedic surgeons (JYK, SWC).

Whether the treatment succeeded or failed was determined at a weekly interval until 4 weeks. Failed resolution was defined as a presence of persistent olecranon bursal fluid collection or swelling recurrence to the initial size at Week 4 after the initiation of the treatment according to the 
Table 2. Treatment outcomes between groups

\begin{tabular}{llll}
\hline Treatment outcome & $\begin{array}{l}\text { Compression/NSAIDs } \\
\text { group }(\mathrm{n}=30)\end{array}$ & $\begin{array}{l}\text { Aspiration group } \\
(\mathrm{n}=26)\end{array}$ & $\begin{array}{l}\text { Aspiration with steroid } \\
\text { injection group }(\mathrm{n}=27)\end{array}$ \\
\hline Resolution (number [\%]) & $25(83 \%)$ & $17(65 \%)$ & $23(85 \%)$ \\
RR* $(95 \%$ CI) & $0.68(0.27-1.72)$ & $2.19(0.98-4.87)$ & $0.59(0.22-1.63)$ \\
Pain VAS & 1.9 (range, 1.3-2.6) & $1.7($ range, $1.0-2.5)$ & $1.7($ range, $1.0-2.5)$ \\
\hline
\end{tabular}

* The relative risk was calculated from the comparison between one group and the other two groups; NSAIDs = nonsteroidal antiinflammatory drugs; $\mathrm{RR}=$ relative risk for failed resolution; $\mathrm{CI}=$ confidence interval; VAS $=$ visual analog scale.

Table 3. Number of resolution according to time

\begin{tabular}{llllll}
\hline Group & Week 1 & Week 2 & Week 3 & Week 4 & Total resolution number \\
\hline Compression/NSAIDs group (number) & 0 & 6 & 9 & 10 & $25 / 30$ \\
Aspiration group (number) & 0 & 4 & 8 & 5 & $17 / 26$ \\
Aspiration with steroid injection group (number) & 6 & 8 & 4 & 5 & $23 / 27$ \\
\hline
\end{tabular}

NSAIDs = nonsteroidal antiinflammatory drugs.

Table 4. Median weeks to resolution of each group

\begin{tabular}{lllll}
\hline Resolution time & Compression/NSAIDs & Aspiration & Aspiration with steroid injection & p value \\
\hline Median weeks to resolution & 3.2 (range, 2-4) & 3.1 (range, 2-4) & 2.3 (range, 1-4) \\
\hline
\end{tabular}

NSAIDs = nonsteroidal antiinflammatory drugs.

previously described protocols. The size of bursal swelling was measured by its length and width at each time. On the contrary, if bursal fluid collection was substantially reduced or completely disappeared by the end of Week 4, the treatment was considered successful.

In addition to assessing resolution, visual analog scales (VASs) were used to evaluate pain (0-10 with 10 defined as the worst) at 4 weeks after the initiation of the treatment. The VAS pain scores included all kinds of pain such as pain at rest, pain with movement, and pain with direct pressure.

\section{Statistical Analysis}

The results were presented as median (range). KruskalWallis test followed by Bonferroni's post hoc analysis was used to evaluate differences among the groups for continuous variables and the chi-square or Fisher's exact test was used for comparison of categorical variables. The relative risk was calculated from the comparison between one group and the other two groups. The primary endpoint was the occurrence of failed resolution after treatment at 4 weeks, and the secondary endpoints were the clinical outcomes in terms of pain VAS and other complications. All statistical analyses were performed using the SPSS software package (Version 18.0; SPSS Inc, Chicago, IL, USA), and a $\mathrm{p}$ value $<0.05$ was considered to represent a statistically significant difference.

\section{Results}

There were no differences in the proportion of patients experiencing resolution among the treatment groups (Group C: 25 of 30 [83\%], relative risk of resolution failure: 0.68 [95\% confidence interval $\{\mathrm{CI}\}, 0.27-1.72], \mathrm{p}=$ 0.580; Group A: 17 of 26 [65\%], relative risk of resolution failure: 2.19 [95\% CI, 0.98-4.87], $\mathrm{p}=0.083$; Group AS: 23 of 27 [85\%], relative risk of resolution failure: 0.59 [95\% CI, 0.22-1.63], $\mathrm{p}=0.398)(\mathrm{p}=0.073$ ) (Table 2). In addition, the pain VAS score showed no difference regardless of the resolution state of olecranon bursitis (mean pain VAS score: 1.7 [1.3-2.1] for the successful resolution group versus 2.2 [1.1-3.2] for the failed resolution group, $\mathrm{p}$ $=0.337$ ) (Table 2).

Steroid injection after aspiration (Group AS) was associated with the earliest resolution (2.3 weeks [range, 1-4 weeks]) when compared with aspiration alone (Group A, 3.1 weeks [range, 2-4 weeks]) and compression bandaging with NSAIDs (Group C, 3.2 weeks [range, 2-4 weeks]), p $=0.015)($ Tables $3,4,5)$. 
Table 5. Multiple comparisons using Bonferroni correction between groups regarding resolution time

\begin{tabular}{lll}
\hline Group & Aspiration group & Aspiration with steroid injection group \\
\hline NSAIDs/compression group & $0.1(-0.6$ to 0.8$), \mathrm{p}>0.999$ & $0.8(0.2-1.5), \mathrm{p}=0.009$ \\
Aspiration group & & $0.7(-0.1$ to 1.4$), \mathrm{p}=0.053$ \\
\hline
\end{tabular}

NSAIDs = nonsteroidal antiinflammatory drugs.

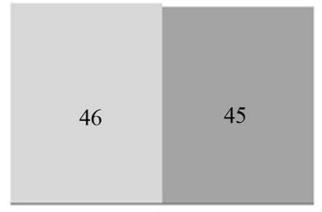

Mean Age (years) $(\mathrm{p}=0.921)$

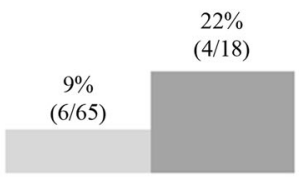

Diabetes

$(\mathrm{p}=0.144)$

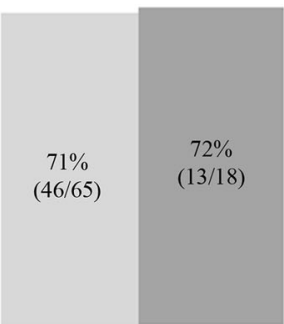

Sex (male)

$(\mathrm{p}=0.578)$

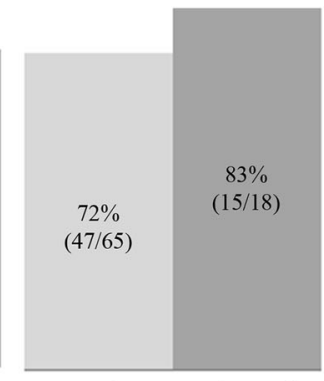

Dominancy of Hand (dominant) $(\mathrm{p}=0.306)$

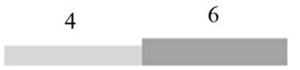

Symptom Duration (weeks) $(\mathrm{p}=0.008)$

Successful Resolution

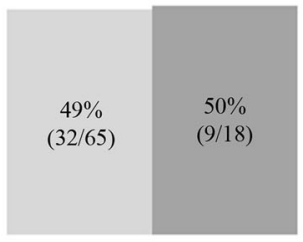

Trauma History $(\mathrm{p}=0.582)$
Failed

Resolution

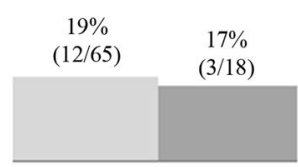

Hypertension $(\mathrm{p}=0.584)$

Fig. 2 Longer duration of symptoms before treatment was the only factor associated with treatment failure by 4 weeks.

Longer duration of symptoms before treatment was the only factor associated with treatment failure by 4 weeks (failed resolution group: 6 weeks [range, 2-9 weeks]; successful resolution group: 4 weeks [range, 0.4-6 weeks]; $\mathrm{p}=0.008$ ). Other factors (age, sex, affected side, history of trauma, diabetes, hypertension) were not associated with treatment failure (Fig. 2).

No complications such as infection, atrophy of the skin in cases with steroid injection, or formation of persistent drainage after aspiration were observed.

\section{Discussion}

Resolution failure after the treatment of nonseptic olecranon bursitis is not an uncommon problem and a challenge for orthopaedic surgeons; however, the study for the effective treatment options for the nonseptic olecranon bursitis was not performed sufficiently until the date of this writing, especially in a randomized prospective study design. The aims of this study were to verify which treatment option has superior results in terms of the highest likelihood of resolution and which brings the earliest resolution among compression bandaging with NSAIDs, aspiration, and aspiration with steroid injection. From this study, we showed that there were no differences in the proportion of patients whose bursitis resolved by Week 4 among the three treatment groups, but the steroid injection after aspiration was associated with the earliest resolution.

Although this is the first study that prospectively compares the outcomes of compression/NSAIDs, aspiration, and aspiration with steroid injection in the treatment of nonseptic olecranon bursitis, several limitations should be pointed out. First, this study was performed at two different hospitals. Accordingly, the patients were treated by two different surgeons (JYK, SWC) and the evaluation of outcomes was also conducted by two different researchers (GYS, JSL). Although aspiration and steroid injection are very simple procedures and the failure of resolution is easy to determine, there may still exist some variations leading 
to errors related to the multicenter study design. Second, the followup of 4 weeks was relatively short. The olecranon bursitis is known to be a condition that commonly recurs, and it may recur even for those patients who seemingly resolved by 4 weeks. The likelihood of differences of resolution among groups might change after 4 weeks followup. Furthermore, other clinical outcomes such as pain VAS and complications might change over a longer period of followup. Third, surgical treatment was not included in the analysis. Because the surgical excision is rarely performed for nonseptic olecranon bursitis [13], we selected only the nonsurgical approaches for the present study. Fourth, the use of NSAIDs for a 1-week period might not be enough to achieve a therapeutic dose against inflammation; therefore, the first week might not be appropriate to time to followup and the prescription of NSAIDs to each treatment arm could have blunted the differences among the groups. Fifth, we identified the nonseptic olecranon bursitis in a clinical basis and we did not perform the analysis of aspirated fluid in patients enrolled in this study because the diagnostic aspiration itself might affect the outcomes. Sixth, we might overestimate the minimal clinically important difference (MCID) and underpowered this study with small sample size calculation. Further study with the MCID less than 30\% based on the result of this study as a pilot data may be needed. In addition, the current study was powered only to examine the primary endpoint, which was the persistent olecranon bursitis at 4 weeks, not to perform the subgroup analyses. Finally, this study was designed to evaluate the treatment efficacy, not the safety; therefore, we might not guarantee the safety of treatment arms of our study as a result of limited sample size, although we could not find any complications such as infection, skin atrophy, or persistent drainage [10].

With regard to resolution of nonseptic olecranon bursitis, the proportion of resolution failure after the treatment of nonseptic olecranon bursitis was reported to range from $19 \%$ to $50 \%[17,19]$, which is in line with the results of the present study (from $15 \%$ to $35 \%$ at 4 weeks, depending on the treatment method). In addition, although several studies reported that the steroid injection after aspiration is an effective and preferred treatment for nonseptic olecranon bursitis compared with NSAID treatment $[2,14,17]$ or aspiration alone treatment [2, 14, 17], we did not detect differences in the proportion of patients whose bursitis resolved among the three treatment groups: compression bandaging with NSAIDs, aspiration, and aspiration with steroid injection. The nonstatistical differences among treatment methods regarding resolution in this study might be the result of the underpowered sample size calculation with a MCID of $30 \%$. However, the rate of resolution failure $(35 \%)$ at 4 weeks with aspiration was unacceptable, especially considering the fact that the corresponding rate was lower in the patients who were treated by compression bandaging with NSAIDs. Although the exact reason behind the high failed resolution of nonseptic olecranon bursitis after aspiration remains to be determined, it can be speculated that aspiration alone cannot eliminate or alleviate the already initiated inflammatory response in the bursa caused by friction of the posterior elbow or its overuse, leading to continuous production of bursal fluid [3]. Moreover, aspiration itself might cause new mechanical injury to the bursa and initiate another inflammatory process, which may explain the higher recurrence rate of nonseptic olecranon bursitis. On the contrary, compression/ NSAIDs may be chosen at ease for the treatment of nonseptic olecranon bursitis because the inflammatory process might subside with time if there is no further damage to the bursa [20]. The relatively low proportion of failed resolution $(17 \%)$ at 4 weeks obtained in Group C supports the efficiency of compression/NSAIDs in the treatment of nonseptic olecranon bursitis. These measures seemed to be sufficient for the resolution of inflammation and resulting decrease in bursal fluid production even if the bursal fluid was not removed intentionally by aspiration.

For the residual pain, the pain VAS assessed at 4 weeks was not different regardless of the resolution or treatment methods. We do not know the exact reason why the failed resolution did not make a difference in the level of pain; however, we think that the mild symptom of nonseptic olecranon bursitis may be a reason. That is, the pain or discomfort of a posterior elbow was mild or negligible in every case of this study (pain VAS of less than 3), and if any, it usually occurred only when the posterior elbow bumped into a hard object inadvertently, which was not common in daily activities in most cases. For this reason, the residual pain did not seem to show any noticeable difference.

Another important finding of this study is the time of resolution. Although the proportions of resolution were not different between the compression/NSAIDs group (Group C) and the steroid injection after aspiration group (Group AS), the AS group showed a slightly faster time to resolution compared with the other groups. Previously, Weinstein et al. [20] observed faster resolution of olecranon bursitis in patients undergoing intrabursal injection of triamcinolone compared with those undergoing initial aspiration and subsequent observation. This rapid and prolonged effect of steroids on bursitis has also been reported for trochanteric bursitis [16] and subacromial bursitis [9]. Therefore, steroid injection after aspiration might be able to be used selectively when rapid resolution of nonseptic olecranon bursitis is required because of associated discomfort or pain or for patients requiring especially rapid convalescence such as athletes and patients 
with high occupational demands [12], although the overall success rate was not different among treatment groups and the long-term outcomes were not confirmed.

We did not detect any complications of treatment, but this study might be too small to detect uncommon complications related to treatment [10]. Moreover, a study of this size cannot draw any inferences about safety nor can it compare the groups in terms of safety [10]. It is important to note that prior studies have amply documented the sometimes severe risks of injections and aspirations of olecranon bursae $[8,17,18,20]$. Based on these concerns, and on the absence of large differences among our study groups, we consider that compression bandaging and short courses of NSAIDs may be the safest approach for these patients and more invasive modalities be reserved for those patients who have failed such treatment $[8,20]$.

In conclusion, with the numbers available, there were no differences in efficacy when compression bandaging with NSAIDs, aspiration, and aspiration with steroid injection were compared. However, we were powered only to detect a $30 \%$ difference, meaning that if there was a smaller difference in efficacy among the groups, we might not have detected it in a study of this size. Our data can be used as pilot data to power future, prospective (and likely multicenter) trials. Because olecranon bursitis can recur, and because treatments like aspiration and aspiration with steroid injection can cause complications, unless future trials demonstrate clear efficacy advantages of aspiration and/or injection both at short and longer terms, we suggest that compression bandaging and a short course of NSAIDs may offer the most appropriate balance of safety and efficacy.

Acknowledgments We thank Ms Ji Hyun Hur and Ye Ji Shin for the support of this study.

\section{References}

1. Abzug JM, Chen NC, Jacoby SM. Septic olecranon bursitis. $J$ Hand Surg. 2012;37:1252-1253.

2. Baumbach SF, Domaszewski F, Wyen H, Kalcher K, Mutschler W, Kanz KG. Evaluation of the current treatment concepts in
Germany, Austria and Switzerland for acute traumatic lesions to the prepatellar and olecranon bursa. Injury. 2013;44:1423-1427.

3. Baumbach SF, Lobo CM, Badyine I, Mutschler W, Kanz KG. Prepatellar and olecranon bursitis: literature review and development of a treatment algorithm. Arch Orthop Trauma Surg. 2014;134:359-370.

4. Cruz C, Shah SV. Dialysis elbow. Olecranon bursitis from longterm hemodialysis. JAMA. 1977;238:243.

5. Degreef I, De Smet L. Complications following resection of the olecranon bursa. Acta Orthop Belg. 2006;72:400-403.

6. Del Buono A, Franceschi F, Palumbo A, Denaro V, Maffulli N. Diagnosis and management of olecranon bursitis. Surgeon. 2012;10:297-300.

7. Garcia-Porrua C, Gonzalez-Gay MA, Ibanez D, Garcia-Pais MJ. The clinical spectrum of severe septic bursitis in northwestern Spain: a 10 year study. J Rheumatol. 1999;26:663-667.

8. Habib GS, Abu-Ahmad R. Lack of effect of corticosteroid injection at the shoulder joint on blood glucose levels in diabetic patients. Clin Rheumatol. 2007;26:566-568.

9. Johansson K, Oberg B, Adolfsson L, Foldevi M. A combination of systematic review and clinicians' beliefs in interventions for subacromial pain. Br J Gen Pract. 2002;52:145-152.

10. Leopold SS. Eitorial: When 'safe and effective' becomes dangerous. Clin Orthop Relat Res. 2014;472:1999-2001.

11. McAfee JH, Smith DL. Olecranon and prepatellar bursitis. Diagnosis and treatment. West J Med. 1988;149:607-610

12. McFarland EG, Mamanee P, Queale WS, Cosgarea AJ. Olecranon and prepatellar bursitis: treating acute, chronic, and inflamed. Phys Sportsmed. 2000;28:40-52.

13. Quayle JB, Robinson MP. A useful procedure in the treatment of chronic olecranon bursitis. Injury. 1978;9:299-302.

14. Rinkel WD, Schreuders TA, Koes BW, Huisstede BM. Current evidence for effectiveness of interventions for cubital tunnel syndrome, radial tunnel syndrome, instability, or bursitis of the elbow: a systematic review. Clin $J$ Pain. 2013;29:1087-1096.

15. Sayegh ET, Strauch RJ. Treatment of olecranon bursitis: a systematic review. Arch Orthop Trauma Surg. 2014;134:15171536.

16. Shbeeb MI, O’Duffy JD, Michet CJ Jr, O'Fallon WM, Matteson EL. Evaluation of glucocorticosteroid injection for the treatment of trochanteric bursitis. J Rheumatol. 1996;23:2104-2106.

17. Smith DL, McAfee JH, Lucas LM, Kumar KL, Romney DM. Treatment of nonseptic olecranon bursitis. A controlled, blinded prospective trial. Arch Intern Med. 1989;149:2527-2530.

18. Stell IM. Management of acute bursitis: outcome study of a structured approach. J R Soc Med. 1999;92:516-521.

19. Stewart NJ, Manzanares JB, Morrey BF. Surgical treatment of aseptic olecranon bursitis. J Shoulder Elbow Surg. 1997;6:49-54.

20. Weinstein PS, Canoso JJ, Wohlgethan JR. Long-term follow-up of corticosteroid injection for traumatic olecranon bursitis. Ann Rheum Dis. 1984;43:44-46. 